\title{
Textual Stylistic Variation: Choices, Genres and Individuals
}

\author{
Jussi Karlgren \\ SICS - Swedish Institute of Computer Science, Box 1263, S-164 29 Kista, Sweden \\ jussi@sics.se
}

Summary. This chapter argues for more informed target metrics for the statistical processing of stylistic variation in text collections. Much as operationalized relevance proved a useful goal to strive for in information retrieval, research in textual stylistics, whether application oriented or philologically inclined, needs goals formulated in terms of pertinence, relevance, and utility — notions that agree with reader experience of text. Differences readers are aware of are mostly based on utility — not on textual characteristics per se. Mostly, readers report stylistic differences in terms of genres. Genres, while vague and undefined, are well-established and talked about: very early on, readers learn to distinguish genres. This chapter discusses variation given by genre, and contrasts it to variation occasioned by individual choice.

\subsection{Stylistic variation in text}

Texts are much more than what they are about. Authors make choices when they write a text: they decide how to organize the material they have planned to introduce; they select amongst available synonyms and syntactic constructions; they target an intended audience for the text. Authors will make their choices in various ways and for various reasons: based on personal preferences, on their view of the reader, and on what they know and like about other similar texts. These choices are observable to the reader in the form of stylistic variation, as the difference between two ways of saying the same thing.

On a surface level this variation is quite obvious, as the choice between items in a vocabulary, between types of syntactical constructions, between the various ways a text can be woven from the material it is made of. A consistent and distinguishable tendency to make some of these linguistic choices can be called a style. It is the information carried in a text when compared to other texts, or in a sense compared to language as a whole. It is not incidental but an integral part of the intended and understood communication, and will impart to the reader a predisposition to understand the meaning of text in 
certain ways. Managing stylistic choice is the mark of a competent author learning how to do so well is a craft which requires training and experience. Recognising stylistic choice is an important component of reading competence - a proficient reader will be sensitive to stylistic variation in texts.

Consistent situationally motivated stragies for making the appropriate stylistic choices is a functional strategy on the part of the author, and enables readers to identify likenesses across individual texts or utterances, thus guiding the reader in understanding them. Such consistent bundled observable occurrence patterns of linguistic items are easily observable and recognisable by statistical analysis and constitute a useful tool for achieving the communicative purposes of the author.

\subsection{Detecting stylistic variation in text}

It is easy enough to establish that there are observable stylistic differences between texts we find in document collections. However, while statistical analysis of stylistic differences between texts is mostly uncomplicated in every particular instance, it is difficult to provide general solutions, without descending into specifics or idiosyncracies. Using the textual, lexical, and other linguistic features we find to cluster the collection — without anchoring information in usage - we risk finding statistically stable categories of data without explanatory power or utility.

A more linguistically informed approach is to start from established knowledge (or established presumption, as it were) and to work with a priori hypotheses on qualities of textual variation. The observations we are able to make from inspection of the linguistic signal are limited by the quality of the analysis tools we have recourse to. The features we find are often on a low level of abstraction and are simultaneously superficial and specific. To abstract to a higher level of abstraction from the observable surface features it is useful to understand them as the exponents of some underlying or latent dimensions of variation within the space of possible texts - Douglas Biber, e.g., whose work has informed much of the work on computational stylistics, has posited dimensions such as Involved vs Informed, Narration vs Argumentation, Personal vs Impersonal.[1, 2]

Investigating such underlying dimensions of variation, establishing hypotheses about which dimensions are reasonable and effective, based on study of the material at hand, on knowledge of human linguistic and communicative behaviour, on understanding of computation and processing constraints, is the main task for computational stylistics today - and a task which only to some extent can be accomplished using purely formal, data-oriented methods, without studying either textuality or readership.

If we wish to contribute to better text handling tools and to a better understanding of human textual practice by the statistical processing of stylistic variation in text collections we need principled hypotheses of human linguistic 
processing and communicative behaviour to base our experimentation on. We need to understand what consequences our claims have on representation, processing, and application, and we need a research methodology which invites the systematic evolution of new results. Much as operationalized relevance has proven a useful goal to strive for in information retrieval, research in textual stylistics, whether application oriented or philologically inclined, needs goals formulated in terms of pertinence, relevance, and usefulness.

Such notions agree with reader experience of text: the differences readers are aware of are mostly based on utility - not on textual characteristics per se.

\subsection{Genres as vehicles for understanding stylistic variation}

Stylistic differences vary by author preferences and by constraints imposed by situation the text is produced in. This is reflected when readers are asked about texts.

In questionnaires or interviews, readers mostly report stylistic differences either by describing the source of the text, or in terms of categories of text, in terms of genres. Readers describe genres in terms utility, in terms of perceived quality of the text in some prescriptive terms, or in terms of complexity of the text and subject matter. On follow-up questioning readers will bring up subjective qualities such as trustworthiness of the text or further discuss readability or other specifics related to complexity, both lexical and syntactic (and specifically making claims in both dimensions simultaneously, typically claiming that leader articles are difficult and long-winded or that sports features are inane and simplistic). Readers will describe genres by describing situations in which the genre is relevant, through experiences of reading a genre, by typical topics or content, and only in some very specific cases do readers give examples of lexical or other linguistic features of the text. [3, e.g.]

Demarcation of stylistic variation to topical variation is of course impossible: the content and form of the message cannot be divorced. Certain meanings must or tend always to be expressed in certain styles: legal matters tend to be written in legal jargon rather than hexameter; car ownership statistics in journalistic or factual style. Drawing a clean distinction between meaning and style, between form an content, is in practice impossible except for certain special cases; it is worth questioning whether there are any formally identifiable styles at all beyond the distinctions that topical analysis already give us $[4$, e.g.].

Genre is a vague but well-established notion, and genres are explicitly identified and discussed by language users. Early on in their reading career, readers learn to distinguish texts of different genres from each other: children's books from encyclopedias, news from magazines, handbooks from novels. Genres 
have a reality in their own right, not only as containers or carriers of textual characteristics.

Genre analysis is a practical tool for the analysis of human activity in many ways in many different fields loosely related to each other, some more formal than other. In recent years, genre analysis has been extended to typologies of communicative situations beyond the purely textual $[5,6$, e.g.], with genres ranging much further than would be afforded by analysis of written text: the range of possible human communicative activities is much wider than the range of possible texts. (This present discussion is mostly concerned with written texts, but it should be noted that generalisations to other communicative situations are obvious and obviously interesting). Each such communicative sphere can be understood to establish its conventions as to how communicative action can be performed. When these conventions bundle and aggregate into a coherent and consistent socially formed entity, they guide and constrain the space of potential communicative expressions and form a genre, providing defaults where choices are overwhelming and constraints no choices should be given.

A text cannot stray too far the prototypical expression expected within the genre, or its readers will be confused and hindered in their task of understanding it. Genre conventions form a framework of recurrent practice for authors and readers alike and a basis of experience within which the communication, its utterances and expressions are understood: participants in the communicative situation use style as a way of economizing their respective communicative effort. This basic premise, that of effective communicative practice on part of participants, should be kept in mind during the study of stylistic variation in text: they will form be the basis on which hypotheses of latent dimensions of appropriate explanatory power can be built.

The perceptions of readers may be interesting, elucidating, and entertaining to discuss, but are difficult to encode and put to practical use. While we as readers are good at the task of distinguishing genres, we have not been intellectually trained to do so and we have a lack of meta-level understanding of how we proceed in the task. To provide useful results, whether for application or for the understanding of human communicative behaviour, research efforts in stylistic analysis must model human processing at least on a behavioural level at least to some extent. We need to be able to address the data on levels of usefulness, and we need to observe people using documents and textual information to understand what is going on.

\subsection{Factors which determine stylistic variation in text}

Stylistic choices are governed by a range of constraints, roughly describable in terms of three levels of variation: firstly, and most obviously observable, choices highly bound and formalized into situation- and text-independent rule 


\begin{tabular}{l|l|l}
\hline Rule & $\begin{array}{l}\text { Language } \\
\text { Convention }\end{array}$ & $\begin{array}{l}\text { Syntax, morphology } \\
\text { genre } \\
\text { Author }\end{array}$ \\
Free & $\begin{array}{l}\text { Lexical patterns, patterns of ar- } \\
\text { gumentation, tropes } \\
\text { Repetition, organisation, elabo- } \\
\text { ration }\end{array}$ \\
\hline
\end{tabular}

Table 8.1. Characteristics of different levels of stylistic constraints.

systems such as spelling, morphology or syntax, taught in schools and explicitly mentioned in lexica, grammars, and writing rules; secondly, choices that are conventional and habitual, bound by context, situation, and genre, mainly operating on the level of lexical choice and phraseology; thirdly, choices on the level of textual and informational organisation where the author of a text operates with the least amount of formal guidance (cf. Table 8.1).

Constraints in the form of language rules are studied by linguists and are understood as obligatory by language users; linguistic conventions, bound by situation are more difficult to pinpoint and formulate, except as guidelines to an author in terms of appropriateness or even "politeness"; constraints on informational organisation are few and seldom made explicit, but are what distinguishes a well-written text from a badly written one.

The conventions that a genre carries are sometimes explicitly formulated, consciously adhered to by authors, and expected by readers. Other times they are implicit and not as clearly enforced. They may even be misunderstood by the parties of a discourse without causing a complete breakdown.

For the purposes of descriptive analysis of texts, genres can be described through a) the communicative purpose which should be somewhat consistent within the set of texts that constitute it; b) the shared and generalised stylistic and topical character of the sets of documents, which also should be somewhat consistent; c) the contexts in which the set of texts appear; d) the shared understanding between author and reader as to the existence of the genre.

This last aspect does not need to be explicit to all readers or even all authors, but in typical cases, the genre is something that can be discussed by experienced authors and experienced readers, and which is intuitive and helpful to both parties. Items belonging to a genre are recognized through their appearance in specific contexts and the presence of some identifiable and characteristic choices. Phrases such as "Once upon a time", "In consideration thereof, each party by execution hereof agrees to the following terms and conditions"; rhyming or alliteration; expressions of personal judgment, opinion and sentiment; explicit mention of dates and locations are all examples of surface cues which are easy to identify. Presentation of texts printed in book form, as hand written pamphlets, read aloud at a fireside or at a lectern in a congress hall are contextual cues that afford us licence to assume them to belong to family of other similar items. 
Establishing a genre palette for further studies can be a research effort in its own right. Understanding how genres are established and how they evolve in the understanding participants have and acquire of a communicative situation is a non-trivial challenge and requires elaborate methodological effort. Examples of such studies are studies of communicative patterns in distributed organisations $[7,8$, e.g.].

However, understanding the social underpinnings of genres is not necessary for most computational approaches. The genres used as target metrics must have some level of salience for their readers and authors, but they must not cover all possible facets and niceties of communication to be useful for the fruitful study and effective application of stylistic variation in text. In many experiments made on computational stylistics, in the case of many experimental collections, and in fact in the minds of many readers, genre has mostly been equated with or based on text source. Since genres are strongly dependent on context, and authorship analysis is one of the prime applications for stylistic analysis, this is a fair approximation. Better approximations than source can be high level categories such as "narrative" vs "expository"; "opinionated" vs "neutral"; "instructional and factual" vs "fiction and narrative" vs "poetry and drama"; or alternatively, specific categories such as "legal", "technical", "commerc ial" as might be found in some specific collection such as a corporate document archive. The central point is that the categories chosen must have some base in usage rather than in statistically determined differences: the latter can be underdetermined and lead the study astray.

\subsection{Individual variation vs situational variation}

Seasoned authors are likely to feel free to break many of the conventions of a genre, in effect creating a voice or style of their own (or even a style specific to some specific text or situation), where a novice author will be more likely to follow conventions, falling back on defaults where experience gives insufficient guidance, using unmarked cases where choice between alternatives is difficult. In this sense, genre gives us a benchmark or a water-line with which to judge individual choice, measuring contrast between functional style, which forms the identifying characteristics of the genre, in contrast with individual style on the level of specific texts or sources. [9]

The span of variation, from variation occasioned by individual performance to that constrained by the expectations given by genre, gives us several targets for stylistic study: we may wish to explore the general characteristics of communicative situations of some specific type, domain or topic; or we may wish to understand the character of a specific author or individual source. After selecting a linguistic item - some lexical item, some construction, some observation - we can study if its occurrence pattern in some sample set of texts varies from the expected occurrence of that specific item (with prior 
information taken into account). This is a mark of individuality and can be used in the process of identifying author, genre, or indeed, topic.

\subsection{Concrete Example: Newsprint and Its Subgenres}

Newsprint, while a special register of human language use in itself, is composed of several well-established subgenres. While newsprint is - together with scientific and intellectual text - over-represented as an object of empirical philological study as compared to other forms of human linguistic communication, it possesses some quite useful qualities for systematical study of linguistic characteristics, chief among them that the texts are most often intended to be the way they are. The textual material has passed through many hands on its way to the reader in order to best conform to audience expectations; individual variation - worth study in itself - is not preserved to any appreciable extent in most cases; the audience is well trained in the genre. In short, the path is well-trod and as such easy to study. Newsprint may sound to be a homogenous mass of text, but it contains several well-established subgenres. In this example one year of Glasgow Herald is studied, and many of the texts are tagged for article type - cf. Table 8.2.

\begin{tabular}{|c|c|}
\hline Article type & $n$ \\
\hline tagged & 17467 \\
\hline advertising 522 & \\
\hline book 585 & \\
\hline correspondence 3659 & \\
\hline feature 8867 & \\
\hline leader 681 & \\
\hline obituary 420 & \\
\hline profile 854 & \\
\hline review 1879 & \\
\hline untagged & 39005 \\
\hline total & 56472 \\
\hline
\end{tabular}

Table 8.2. Sub-genres of the Glasgow Herald.

It is easy enough to establish that there are observable differences between the genres we find posited in the textual material. Trawling the (morphologically normalized) texts for differences, comparing each identified category in Table 8.2 with the other categories we find for a one month sample of text some of the most typical words for each category as per Table 8.3. "Typical" is here operationalized as words with a document frequency deviating from 
expected occurrence as assessed by $\chi^{2}$. This sort of analysis can be entertaining, at times revealing, but cannot really give us any great explanatory power. Even the handful of example terms shown in Table 8.3 are clearly coloured by the subject matter of the sub-genres and only to some extent reveal any stylistic difference between them.

\begin{tabular}{|c|}
\hline Article type Typical words \\
\hline advertising provide, available, service, \\
specialist, business \\
book review novel, prose, author, \\
literary, biography, write \\
correspondence (Various locations in Scotland), \\
overdue, SNP \\
feature \\
say, get, think, put, there, \\
problem, tell \\
leader evident, government, outcome, \\
opinion, even \\
church, wife, daughter, \\
survive, former \\
profile \\
recall, career, experience, \\
musician \\
review concert, guitar, piece, \\
beautifully, memorable
\end{tabular}

Table 8.3. Typical words in sub-genres of one month of the Glasgow Herald.

\subsection{Measurements and Observanda}

Measurement of linguistic variation of any more sophisticated kind has hitherto been hampered by lack of useful tools. Even now, most measurements made in text categorisation studies, be it for authorship attribution, topical clustering, readability analysis or the like, compute observed frequencies of some lexical items, or some identifiable constructions. These measurements are always local, inasmuch they only take simple appearances of some linguistic item into account. Even when many such appearances are combined into an average or an estimate of probability of recurrence, the measurement in question is still local, in the sense that they disregard the structure and progression of the communicative signal. An observed divergence in a text sample from the expected occurrence rate of that specific item (with prior 
information taken into account) is a mark of specific character and can be used in the process of identifying e.g. topic, genre, or author.

The examples given above in Table 8.4 are of this kind. A systematic tendency to pursue certain choices on the local level may be symptomatic of an underlying dimension, but is hardly likely to have any more far-reaching explanatory or predictive power. It is difficult to systematically explore, find, and formulate sensible or adequate dimensions on any level of abstraction above the trivial if the features we have at our disposal are limited to average occurrence frequency of pointwise observations of simple linguistic items.

Textual variation should be measured on the levels on which authors and readers process information, not on the level of whitespace separated character strings. Tentative candidates for more textual measurements could be using term recurrence rather than term frequency [10]; term patterns [11]; type-token ratio [12]; rhetorical structure; measures of textual cohesion and coherence; measures of lexical vagueness, inspecificity, and discourse anchoring; and many other features with considerable theoretical promise but rather daunting computational requirements.

Leaving the simple lexical occurrence statistics and reaching for features with better informational potential proves to be rather unproblematic. We know from past studies and judicious introspection that interview articles contain quotes and that leader articles contain argumentation; both are to be expected to contain pronouns and overt expressions of opinion. Table 8.4 shows some such measurements - with indicated significance (better than 95\%) assessed for each sub-genre compared to the entire collection by MannWhitney U. The explanatory power of this table - again, while interesting in some respects and worth discussion - is rather low, and application of the findings for prediction of genre for unseen cases will be unreliable. A more abstract level of representation is necessary to be able to extract useful predictions and to gain understanding of what textual variation is about.

\subsection{Aggregation of Measurements}

A text will typically yield a number of observations of a linguistic item of interest. Most experiments average the frequency of observations and normalise over a collection of texts, or calculate odds and compare to estimated likelihoods of occurrence, as the example given above. Aggregating observations of linguistic items by averaging local occurrence frequencies over an entire text does not utilise the specific character of text, namely that it is a non-arbitrary sequence of symbols.

There is no reason to limit oneself to pointwise aggregation of observations: the inconvenience of moving to other aggregation models is minimal. In another experiment using the same data as above we have shown that text configurational aggregation of a feature yields better discriminatory power than pointwise aggregation does [14]. 


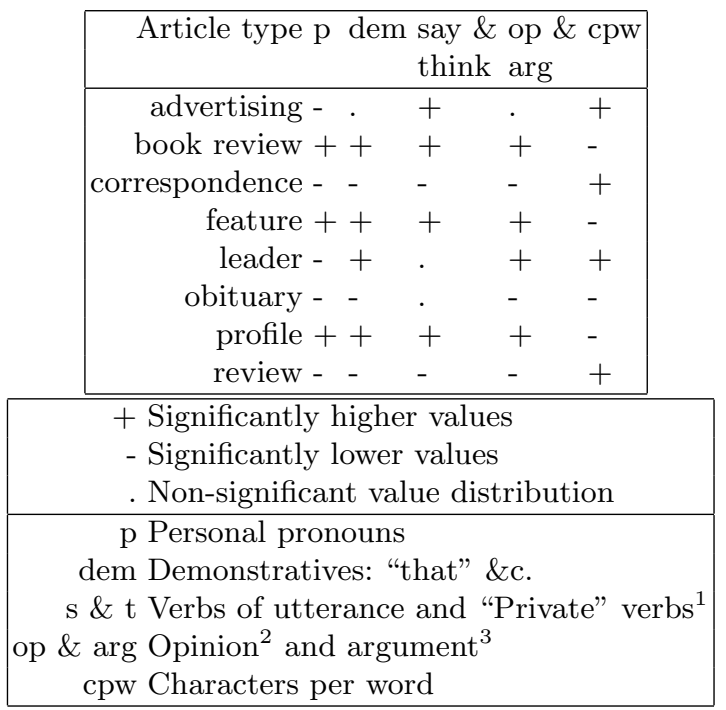

Table 8.4. Some measurements of linguistic items per sub-genre of one year of the Glasgow Herald.

To obtain simple longitudinal patterns each observed item is measured over sliding windows of one to five sentences along each text, and the occurrence of the feature is recorded as a transition pattern of binary occurrences, marking the feature's absence or presence in the sentences within the window. The first and last bits of text where the window length would have extended over the text boundary can be discarded. The feature space, the possible values of the feature with a certain window size is thus all the possible transition patterns for that window size. For windows of size two, the feature space consists of four possible patterns, for windows of size five, thirty-two, as shown in Table 8.5.

\subsection{Concrete example: Configurational features}

Our hypothesis is that author (and speaker) choice on the level of informational structuring and organisation (cf., again, Table 8.1) is less subject to pressure from conventionalisation and grammaticalisation processes ${ }^{4}$. This both by virtue of wide scope, which limits the possibilities of observers to track usage, as well as the many degrees of freedom open for choice, which makes rule expression and rule following inconvenient.

\footnotetext{
${ }^{4}$ This experiment is reported in full in [14]. This section is an excerpt of that paper.
} 


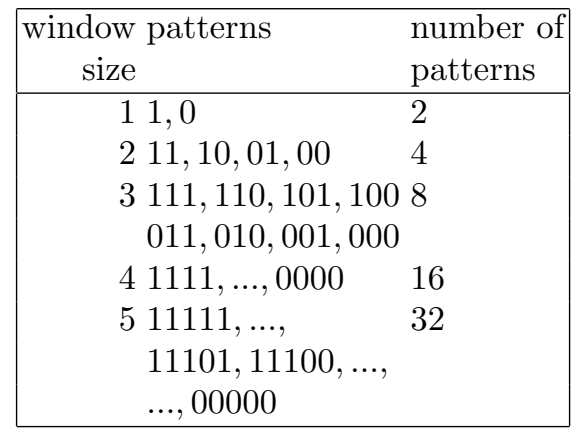

Table 8.5. Feature space for varying window size.

We believe that configurational aggregation of observations might improve the potential for categorisation of authors, since they preserve some of the sequential information which is less constrained by convention and rules. The experiment is designed to investigate whether using such longitudinal patterns improves the potential for author identification more than it improves the potential for genre identification: these transition patterns can then be compared for varying window lengths - the operational hypothesis being that a longer window length would better model variation over author rather than over genre.

Using the same data from Glasgow Herald, we calculated a simple binary feature, noting the occurrence of more than two clauses of any type in a sentence. Each sentence was thus given the score 1 or 0 , depending on whether it had several clauses or only one. This feature is a somewhat more sophisticated proxy for syntactic complexity than the commonly used sentence length measure.

The measurements are given in Table 8.6 for all genres, and the authors with the highest and lowest scores for each variable. As seen from the table, genres are more consistent with each other than are authors: the range of variation between 0.52 to 0.96 is greater than that between 0.78 and 0.93 .

Expanding the feature space four-fold the relative presence for the various sequences of multi-clause sentences, in a window of size two, are shown in Table 8.7 for the genres labeled in the data set and for some of the more prolific authors.

This table is difficult to compare to the measurements in Table 8.6, but using the frequency counts as probability estimates, we can use Kullback-Leibler divergence measure[15] as a measure of differences between measurements. The Kullback-Leibler divergence is a measure of distance between the states in the probability distribution, a large divergence indicating better separation between states - which is desirable from the perspective of a categorisation 


\begin{tabular}{|cc|}
\hline \multicolumn{2}{|c|}{ category multi-clause $\left(f_{1}\right)$ single-clause $\left(f_{0}\right)$} \\
\hline advertising 0.90 & 0.10 \\
book 0.83 & 0.17 \\
correspondence 0.92 & 0.08 \\
feature 0.93 & 0.07 \\
leader 0.93 & 0.07 \\
obituary 0.78 & 0.22 \\
profile 0.92 & 0.08 \\
review 0.87 & 0.13 \\
\hline author $A_{M} 0.96$ & 0.04 \\
author $A_{m} 0.52$ & 0.48 \\
\hline
\end{tabular}

Table 8.6. Relative presence of multi-clause feature "clause" in sentences.

task, since that would indicate better potential power for working as a discriminating measure between the categories under consideration. Since the measure as defined by Kullback and Leibler is asymmetric, we here use a symmetrised version, a harmonic mean given by [16]. In this experiment, with eight genre labels and several hundred authors, we perform repeated resampling of eight representatives, fifty times, from the set of authors and average results to obtain comparable results with the genre distribution.

For each window length, the sum of the symmetrised Kullback-Leibler measure for all genres or authors is shown in Figure 8.8. The figures can only be compared horizontally in the table - the divergence figures for different window sizes (the rows of the table), cannot directly be related to each other, since the feature spaces are of different size. This means that we cannot directly say if window size improves the resulting representation or not, in spite of the larger divergence values for larger window size. We can, however, say that the difference between genre categories and author categories is greater for large window sizes. This speaks to the possibility of our main hypothesis holding: a larger window size allows a better model of individual choice than a shorter one.

\subsection{Conclusion: Target Measures}

Our example experiment shows how the choice of observed linguistic item, choice of aggregation method, and choice of target hypothesis all work together towards making sustainable statements about stylistic variation.

The experiment shows that one method of aggregation gives different results from another set; they also show that modelling author behaviour can profitably be modelled by different features than genre - presumably, in the one case, identifying conventions, in the other, avoiding them. 


\begin{tabular}{|r|llll|}
\hline genre & $f_{11}$ & $f_{10}$ & $f_{01}$ & $f_{00}$ \\
\hline feature & 0.022 & 0.078 & 0.056 & 0.84 \\
review & 0.041 & 0.13 & 0.072 & 0.76 \\
advertising & 0.011 & 0.072 & 0.039 & 0.88 \\
profile & 0.016 & 0.056 & 0.040 & 0.89 \\
leader & 0.016 & 0.055 & 0.023 & 0.91 \\
correspondence & 0.066 & 0.15 & 0.051 & 0.73 \\
obituary & 0.0079 & 0.072 & 0.023 & 0.90 \\
book & 0.038 & 0.084 & 0.069 & 0.81 \\
\hline author & $f_{11}$ & $f_{10}$ & $f_{01}$ & $f_{00}$ \\
\hline$A_{1}$ & 0.013 & 0.071 & 0.052 & 0.86 \\
$A_{2}$ & 0.021 & 0.050 & 0.018 & 0.92 \\
$A_{3}$ & 0.018 & 0.11 & 0.088 & 0.78 \\
$A_{4}$ & 0.19 & 0.097 & 0.032 & 0.68 \\
$A_{5}$ & 0.013 & 0.11 & 0.052 & 0.82 \\
$A_{6}$ & 0.0062 & 0.071 & 0.020 & 0.90 \\
$A_{7}$ & 0.018 & 0.063 & 0.038 & 0.88 \\
$A_{8}$ & 0.0067 & 0.047 & 0.032 & 0.91 \\
$A_{9}$ & 0.010 & 0.064 & 0.027 & 0.90 \\
\hline
\end{tabular}

Table 8.7. Relative frequency of multi-clause sentence sequences for genres and some authors, window size 2 .

\begin{tabular}{r|ll}
\hline Window size & Genre Author \\
\hline 1 & 0.5129 & 0.7254 \\
2 & 0.8061 & 1.3288 \\
3 & 1.1600 & 2.1577 \\
4 & 1.4556 & 2.3413 \\
5 & 1.7051 & 3.0028 \\
\hline
\end{tabular}

Table 8.8. Window length effect.

In general, only if useful target measures of pertinence, relevance, and utility can be found for evaluation, couched in terms derived from study of readership and reader assessment of texts for real needs can we hope to accomplish anything of lasting value in terms of understanding choice, textual variation, and reading. This, in turn, can only be achieved beginning from an understanding that stylistic variation is real on a high level of abstraction, in the sense that readers, writers, editors all are aware of genres as an abstract category; that genres are a useful mid-level category to fix an analysis upon, and that there is no independent non-arbitrary genre palette outside the communicative situation the study is modelling. 
Results of any generality can only be achieved with more informed measures measures of variation and choice, couched in terms of linguistic analysis rather than processing convenience, and aggregated by computational models related to the informational processes of the author and reader.

\section{References}

1. Biber, D.: Variation across speech and writing. Cambridge University Press, Cambridge, UK (1988)

2. Biber, D.: A typology of english texts. Linguistics 27, 3-43 (1989)

3. Dewe, J., Karlgren, J., Bretan, I.: Assembling a balanced corpus from the internet. In: Proceedings of the 11th Nordic Conference of Computational Linguistics. University of Copenhagen, Copenhagen, Denmark (1998)

4. Enkvist, N.E.: Linguistic Stylistics. Mouton, The Hague, Netherlands (1973)

5. Bakhtin, M.M.: The problem of speech genres. In: Speech Genres and Other Late Essays. University of Texas Press, Austin, Texas (1986). Translated by Vern W. McGee

6. Swales, J.: Genre Analysis: English in Academic and Research Settings. Cambridge University Press, Cambridge, UK (1990)

7. Yates, J., Orlikowski, W.J.: Genres of organizational communication: A structurational approach to studying communication and media. Academy of Management Review 17, 299-326 (1992)

8. Orlikowski, W.J., Yates, J.: Genre repertoire: Examining the structuring of communicative practices in organizations. Adminstrative Science Quarterly 39, 541-574 (1994)

9. Vachek, J.: Some remarks on functional dialects of standard languages. In: H. Ringbom (ed.) Style and Text — Studies presented to Nils Erik Enkvist. Skriptor, Stockholm, Sweden (1975)

10. Katz, S.: Distribution of content words and phrases in text and language modelling. Natural Language Engineering 2, 15-60 (1996)

11. Sarkar, A., de Roeck, A., Garthwaithe, P.H.: Term re-occurrence measures for analyzing style. In: Textual Stylistics in Information Access. Papers from the workshop held in conjunction with the 28th International Conference on Research and Development in Information Retrieval (SIGIR). ACM SIGIR, Salvador, Brazil (2005)

12. Tallentire, D.: Towards an archive of lexical norms: A proposal. In: A. Aitken, R. Bailey, N. Hamilton-Smith (eds.) The Computer and Literary Studies. Edinburgh University Press (1973)

13. Quirk, R., Greenbaum, S., Leech, G., Svartvik, J.: A comprehensive grammar of the English language. Longman, London, England (1985)

14. Karlgren, J., Eriksson, G.: Authors, genre, and linguistic convention. In: Proceedings of the SIGIR Workshop on Plagiarism Analysis, Authorship Identification, and Near-Duplicate Detection. Amsterdam (2007)

15. Kullback, S., Leibler, R.A.: On informmation and sufficiency. Annals of Mathematical Statistics 22, 79-86 (1951)

16. Johnson, D.H., Sinanović, S.: Symmetrizing the kullback-leibler distance. IEEE Transactions on Information Theory (2001) 